\title{
A Swarm Intelligent Algorithm Based Route Maintaining Protocol for Mobile Sink Wireless Sensor Networks
}

\author{
Xiaoming $\mathrm{Wu} \mathrm{u}^{1,2,3}$ Yinglong Wang, ${ }^{1,2,3}$ and Yifan $\mathrm{Hu}^{2,3}$ \\ ${ }^{1}$ College of Information Science and Engineering, Shandong University of Science and Technology, Qingdao 266590, China \\ ${ }^{2}$ Shandong Computer Science Center (National Supercomputer Center in Jinan), Jinan 250014, China \\ ${ }^{3}$ Shandong Provincial Key Laboratory of Computer Network, Jinan 250014, China \\ Correspondence should be addressed to Yinglong Wang; wangyl@sdas.org
}

Received 2 June 2015; Revised 5 September 2015; Accepted 30 September 2015

Academic Editor: Yuan Fan

Copyright (C) 2015 Xiaoming Wu et al. This is an open access article distributed under the Creative Commons Attribution License, which permits unrestricted use, distribution, and reproduction in any medium, provided the original work is properly cited.

Recent studies have shown that mobile sink can be a solution to solve the problem that energy consumption of sensor nodes is not balanced in wireless sensor networks (WSNs). Caused by the sink mobility, the paths between the sensor nodes and the sink change frequently and have profound influence on the lifetime of WSN. It is necessary to design a protocol that can find efficient routings between the mobile sink and nodes but does not consume too many network resources. In this paper, we propose a swarm intelligent algorithm based route maintaining protocol to resolve this issue. The protocol utilizes the concentric ring mechanism to guide the route researching direction and adopts the optimal routing selection to maintain the data delivery route in mobile sink WSN. Using the immune based artificial bee colony (IABC) algorithm to optimize the forwarding path, the routing maintaining protocol could find an alternative routing path quickly and efficiently when the coordinate of sink is changed in WSN. The results of our extensive experiments demonstrate that our proposed route maintaining protocol is able to balance the network traffic load and prolong the network lifetime.

\section{Introduction and Related Work}

Wireless sensor network (WSN) is an intelligent monitoring self-organized network consisting of many microsensor nodes with the capabilities of communication, sensing, and computing deployed inside or around the monitoring area. It has broad application prospects and great application value in industrial and agricultural control, urban management, environmental testing, hazardous area remote control, and other fields. WSN involves multiple frontier research fields; it is considered as one of the top-ten world-changing technologies in the future [1].

A typical WSN is composed of many sensor nodes and one or several sinks. The sink collects data from the sensing environment. However, if the sink involved in collecting data is static, the sensors connected directly to a sink deplete their energy much faster than the rest of the network since they carry all the data gathered by the sensors [2], which is called "the crowded center effect" [3] or "energy hole problem" $[4,5]$. One method to avoid the formation of energy holes is to use sink mobility. The sensor nodes can take turns to become the neighbors of the sink due to the sink mobility in a controlled manner, so the energy is consumed evenly among the nodes. It has been demonstrated that a mobile sink can potentially prolong the network's lifetime as the mobile sink would cause the sensor nodes to consume less energy [6].

Mobile sink can be a solution to solve the problem that energy consumption of nodes is not balanced in WSN; we call it mobile sink WSN (MSWSN); the network architecture is illustrated in Figure 1; the source nodes constantly deliver data through multihops relay node path to the mobile sink. But caused by the sink mobility, the paths between the sensor nodes and the sink would change with time [7]. Routing protocol in MSWSN is a great challenge due to the following reasons. Firstly, it is not easy to grasp the whole network topology and it is hard to find a routing path. Secondly, sensor nodes are tightly constrained in terms of energy, processing, and storage capacities. The unpredictable and constant changes in the sink's location form the obstacle of designing the route maintaining protocols in the energy 


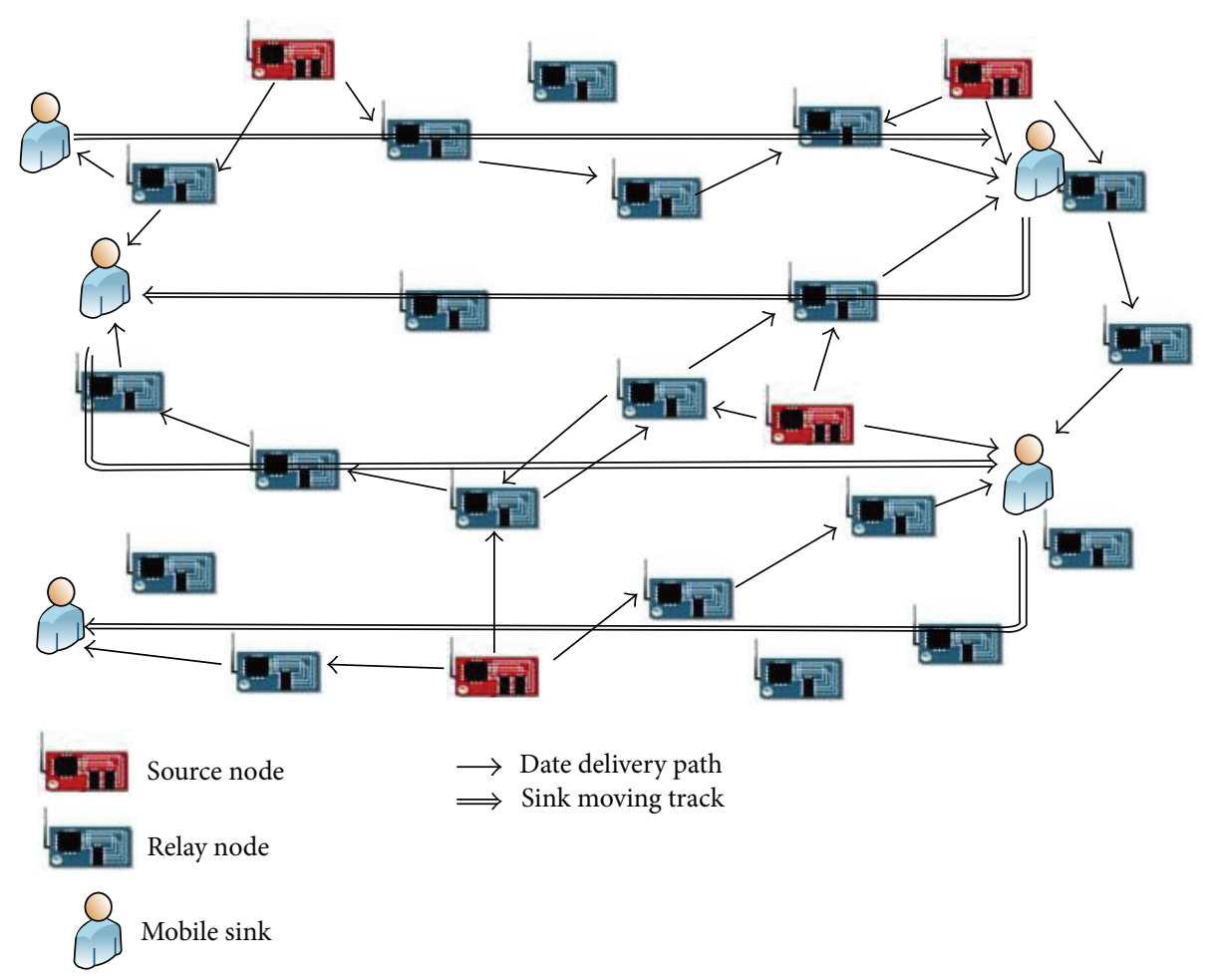

FIGURE 1: Mobile sink wireless sensor network model.

constrained WSN [8]. It is necessary to design a protocol that can find efficient routes between the mobile sink and sensor nodes but does not consume too many network resources.

Various routing protocols have been proposed to address the routing problem of mobile sink. In the literature [9], the TTDD concentrates on efficient data delivery to mobile sinks by clustering nodes into cells. Mobile sinks broadcast request packets only in their local cell, and an overlay routing scheme keeps track of the current cells of the sinks for routing data to them. While effective in high mobility scenarios, the overhead to build and maintain the overlay is significant, especially in periodic reporting scenarios, which are more traffic intensive than event-based reporting. Thus, TTDD is better suited to event-detecting sensor networks with sporadic rather than continuous traffic.

SEAD [10] and its developed protocol DEED [11] attempt to optimize routes from a single source to mobile sinks by allowing each sink to select an access sensor node. A data delivery tree is built between the source and all access nodes based on a geographic location heuristic. When the sink moves, a path between its current nearest neighbor and the access node is maintained, eliminating the need to rebuild the tree. However, if the sink moves far away, a new access node would be selected and the tree is rebuilt.

An enhanced real time with load distribution (ERTLD) routing protocol for mobile WSN is proposed in literature [12]. ERTLD utilizes corona mechanism and optimal forwarding metrics to forward the data packet in mobile WSN. It ensures high packet delivery ratio and experiences minimum end-to-end delay and enhances the total performance, reliability, and flexibility of data forwarding mechanism in mobile WSN.

However, in these classical routing protocols, the route maintaining scheme for the large scale sensor nodes has rarely been considered. Moreover, as the routing optimization problem to find the optimal routing is a NP-hardness problem, the heuristic deterministic methods always fall into local optimum and only get the approximate optimal result. In this paper, we propose a swarm intelligent algorithm optimized route maintaining protocol to optimize the routing path of the MSWSN, and this optimization algorithm would converge to the optimal resolution of the path. Our proposed scheme is developed on the basis of the corona mechanism in ERTLD and differs from the above works. The main contributions of this paper are as follows:

(1) We utilize the concentric ring mechanism to guide the route researching direction and adopt self-adapt feature of our swarm intelligent routing protocol to deal with the dynamic route maintaining problem of MSWSN, which results in saving energy and maximizing packet delivery ratio.

(2) We analyze and compare our protocol with TTDD [9] and ERTLD [12] in terms of packet delivery and energy consumption. Simulation results show superiority of the protocol. 


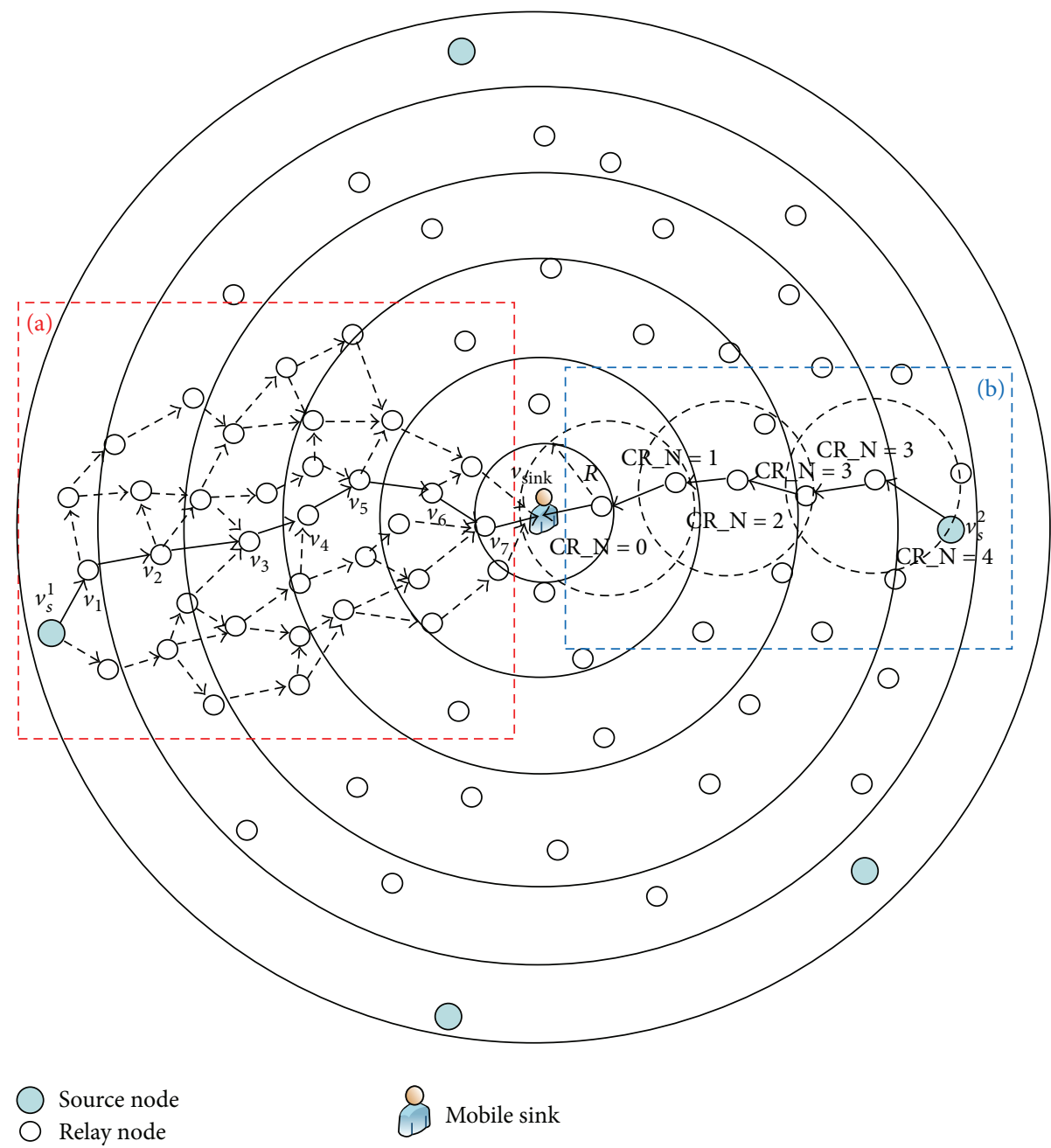

FIGURE 2: The route maintaining model of MSWSN. (a) The process of delivering route maintaining packet through possible paths. (b) The concentric ring number of each node on the path.

The contributions of this paper are different from the literatures $[8,13]$, though the network models of them are all the WSNs with mobile sink:

(1) The routing mechanism is different, we provide concentric ring mechanism to detect the relative distance and direction of each node to the mobile sink, in order to efficiently guide the route researching direction from source node to the sink, comparing to the routing researching mechanism without direction guidance in [13] and another different routing mechanism in [8].

(2) The kernel optimization algorithm of routing protocol is different; our protocol adopts the artificial bee colony $(\mathrm{ABC})$ algorithm to optimize the forwarding path, comparing to the particle swarm optimization algorithm in literature [13] and machine learningbased approach in literature [8]. The tests for several standard functions by [14] have shown that the performance of $\mathrm{ABC}$ is better than the other populationbased algorithms with the advantage of employing fewer control parameters, such as PSO and other algorithms.

The rest of this paper is organized as follows. Section 2 formulates our network model and method. Section 3 describes the proposed algorithm in detail, and the routing protocol is analyzed. Section 4 evaluates the performance of the IABC by comparing it with other routing protocols. Finally, the conclusion is presented in Section 5.

\section{Description of Network Model}

2.1. Concentric Ring Mechanism. The MSWSN is modeled as a time-dependent connected graph $G(V, E)$, where $V$ is a finite set of sensor nodes and $E$ is the set of edges representing connection between these nodes. The mobile sink is a rendezvous point and moves in the network area for the purpose of collecting information of nodes. In our model, the regular moving track of the mobile sink is the rectangular polyline track, as shown in Figure 1, which can efficiently traverse the whole network. The similar traverse track method has been used in the literature [15]. Figure 2 
represents a certain time $T_{0}$ when the mobile sink has moved to place A in the network model. $v_{i}(0<i<N-2)$ is the relay sensor node, $v_{s}$ is the source node, and $v_{\text {sink }}$ is the mobile sink. Suppose there exist $m(0<m<N-1)$ source nodes and one mobile sink; these source nodes can route the packets through multihops relay node path to the mobile sink, as illustrated in the right blue dash-line box (b) of Figure 2. After deploying in the middle area of the network, the mobile sink would broadcast the concentric ring (CR) packets to its one-hop neighbors, in order to determine the concentric ring number for all sensor nodes in the network. The main fields of CR are CR_N (the ring number; initial number is zero) and CR_ID (the packet ID sent by sink). Then, the nodes forward the packet to the next-hop neighbors. The sink would not rebroadcast the CR packet until it moves to a new coordinate which is more than $D$ meters away from the previous coordinate. In our assumption, if the moving distance of sink is less than $D$ meters, it is still within the communication range of the closest relay node.

As shown in Figure 2, all circles are concentric at the sink and can form the coordinate system, and the width of each concentric ring is assumed to be equal to the sensor node's transmission range $R$. Therefore, each sensor node would belong to exactly one concentric ring. The data would be delivered from node $v_{i}$ with high value of CR_N to node $v_{j}$ with low value of CR_N and at last to the mobile sink. If there is not any candidate with lower number of CR_N than $v_{i}$ in the neighbor table of $v_{i}$, data would be forwarded to the node with the same number of CR_N. The right blue box (b) of Figure 2 shows the CR_N of each node on the multihop path.

When the mobile sink moves to the random position, the coordinate system is also changed. Once the sink moves up to $R$ meters away, it would rebroadcast the CR_N to the network, and each node's CR_N would be changed. The packet CR would be delivered to all one-hop neighbors. If one relay node receives CR, it would check CR_ID; if it is the same as the node's previous CR_ID, the node would discard the packet; if not, the node would increase CR_N in CR and save this value as its new concentric ring number. Then, the node would broadcast CR to its neighbors, until the nodes of the whole network are upgraded. With this method, the network can automatically respond to the dynamic topology. Once the sink moves to a certain distance, the previous scenario would be repeated. Using the concentric ring mechanism, the relative distance and direction of each node to the mobile sink can be detected, and the following routing research from source node can be guided toward the direction of sink.

Our network routing model is based on the following assumptions: (1) The area is covered by a large number of homogeneous sensor nodes. Sensor nodes are stationary, but sink moves and changes position constantly with a relatively fixed speed. (2) Data is sensed and transmitted from each source node to the mobile sink every $T$ time period.

2.2. Route Maintaining Algorithm. The concentric ring mechanism can calculate the sensor node's ring number according to its distance to the sink. The following routing algorithm can calculate and decide the optimal forwarding path from the source nodes to the mobile sink, in order to update and maintain the dynamic route.

As assumed, the path generated from a given source node $v_{s}^{1}$ to the mobile sink $v_{\text {sink }}$ is denoted by $p_{j}(j \in 1$, $2, \ldots, n)$. The $k$ th relay node on path $p_{j}$ is denoted by $v_{j}^{k},\{k \in$ $\left.1,2, \ldots, h_{j}\right\}$, in which $h_{j}$ is the hop count on path $p_{j} . e_{j}^{m}$ represents the $m$ th direct edge between two neighbor nodes on $p_{j}$. Let $N\left(p_{j}\right)=\left\{v_{j}^{S}, v_{j}^{1}, v_{j}^{2}, \ldots, v_{j}^{k}, \ldots, v_{j}^{n}\right\} \subset N(v)$ be the set of the sensor nodes existing along path $p_{j}$, where $k$ represents the distance from the sink to the node on a hop scale. As shown in the right blue box (b) of Figure 2, path $p_{1}$ connects source $v_{s}^{1}$ with the sink and contains 6 relay nodes $\left\{v_{1}^{1}, v_{1}^{2}, v_{1}^{3}, v_{1}^{4}, v_{1}^{5}, v_{1}^{6}\right\}$, and 7 relay edges $\left\{e_{1}^{1}, e_{1}^{2}, e_{1}^{3}, e_{1}^{4}, e_{1}^{5}, e_{1}^{6}, e_{1}^{7}\right\}$. Each node $v$ owns its neighbor table, which stores its surrounding neighbor node's ID $v_{i}$ and other information (node signal strength $\operatorname{RSSI}\left(v_{i}\right)$ from the next-hop node $v_{i}$, end-to-end delay $\operatorname{Delay}\left(v_{i}\right)$ between the node and $v_{i}$ ).

The movement of mobile sink would firstly upgrade all the nodes' CN_R. Once the sink has moved out of the communication distance of relay node $v_{j}^{n}$ and the data packet cannot be delivered to the sink, the routing failed information packet would be sent backward to the source node $v_{j}^{s}$ through path $p_{j}$; then, the route maintaining algorithm would be implemented.

$v_{j}^{s}$ would broadcast the route maintaining $(\mathrm{RM})$ packets to one-hop neighbors, and the packet contains the information of battery voltage and source's CR_N (called CR_N_S). Our rule is that if the relay node's CR_N is not higher than the source's CR_N_S, it can relay RM packet, so RM can be delivered forward of the mobile sink. If the relay node $v_{i}$ receives $\mathrm{RM}$, it will check its own $\mathrm{CR} \_\mathrm{N}$; if its $\mathrm{CR}_{-} \mathrm{N}$ is higher than CR_N_S in RM, $v_{i}$ will discard the packet; if not, $v_{i}$ will decrease and save CR_N_S in RM and relay RM to its onehop neighbors; the packet would contain information of its $\operatorname{RSSI}\left(v_{i}\right)$, Delay $\left(v_{i}\right)$, and its battery voltage $V_{\text {bat }}\left(v_{i}\right)$. Then, the node will relay RM to the other neighbors, until the data packet RM is delivered through any multihop path $p_{j}(0<$ $j<n)$ to the mobile sink. Thus, the sink would receive several packets RM from these paths, and all the paths delivering $\mathrm{RM}$ to the sink are considered as the possible alternative paths set $P_{\text {all }}$. The process of delivering packet RM through multihop paths is illustrated in the left red dash-line box (a) of Figure 2.

With this method, the optimal path $p_{\text {op }}$ with the suitable relay node sequence $\left\{v_{\text {op }}^{S}, v_{\text {op }}^{1}, v_{\text {op }}^{2}, \ldots, v_{\text {op }}^{k}, \ldots\right.$, $\left.v_{\text {op }}^{n}\right\}$ is among these possible alternative paths set $P_{\text {all }}$. The sink collects all the information and extracts $\operatorname{RSSI}\left(v_{i}\right)$, $\operatorname{Delay}\left(v_{i}\right)$, and $V_{\mathrm{bat}}\left(v_{i}\right)$ of these nodes on the possible alternative paths $P_{\text {all }}$ and calculates the fitness value of path and selects one optimal alternative path $p_{\text {op }}$ with optimal fitness fitness $\left(p_{\text {op }}\right)$ (e.g., path $\left\{v_{S}^{1}, v_{1}, v_{2}, v_{3}, v_{4}\right.$, $\left.v_{5}, v_{6}, v_{7}\right\}$ in left red box (a) of Figure 2). The nodes sequence of a possible alternative path $p_{j}$ is $\left\{v_{j}^{S}, v_{j}^{1}, v_{j}^{2}, \ldots, v_{j}^{k}\right.$, $\left.\ldots, v_{j}^{n}\right\}$, and the factors affecting the choice of $p_{j}$ include the 
nodes' signal strength, end-to-end delay, and battery voltage on the path. These parameters can determine the fitness function of $p_{j}$, fitness $\left(p_{j}\right)$ :

$$
\begin{aligned}
& \text { fitness }\left(p_{j}\right)=\max \left(\lambda_{1} \sum_{i=1}^{n} \frac{\operatorname{RSSI}_{\mathrm{th}}}{\operatorname{RSSI}\left(v_{j}^{i}\right)}\right. \\
& \left.+\lambda_{2} \sum_{i=1}^{n} \frac{\text { Delay }_{\text {th }}-\operatorname{Delay}\left(v_{j}^{i}\right)}{\text { Delay }_{\text {th }}}+\lambda_{3} \sum_{i=1}^{n} \frac{V_{\text {bat }}\left(v_{j}^{i}\right)}{V_{\text {batth }}}\right),
\end{aligned}
$$

where $\operatorname{RSSI}\left(v_{j}^{i}\right)$ is the signal strength of $v_{j}^{i}$ from the nexthop node $v_{j}^{i-1}$ and its value can indirectly reflect the distance between $v_{j}^{i}$ and $v_{j}^{i-1}$. RSSI $\mathrm{R}_{\mathrm{th}}$ is the signal strength value at threshold point $1 \mathrm{~m}$ which is $-45 \mathrm{dBm}$. Delay $\left(v_{j}^{i}\right)$ is the endto-end delay between the node and next-hop node; Delay ${ }_{\text {th }}$ is the end-to-end delay threshold which is set to $250 \mathrm{~ms} . V_{\text {bat }}\left(v_{j}^{i}\right)$ is the node battery voltage; its value can indirectly reflect the remaining energy of $\left(v_{j}^{i}\right) . V_{\text {batth }}$ is the node threshold battery voltage and equals $3.5 \mathrm{v} . \lambda_{1}, \lambda_{2}$, and $\lambda_{3}$ are the weights of remaining energy, delay, and distance constraints in the fitness function, respectively, and $\lambda_{1}+\lambda_{2}+\lambda_{3}=1$. We set $\lambda_{1}=0.4, \lambda_{2}=0.2$, and $\lambda_{3}=0.4$. The higher fitness value indicates the more suitable routing path; therefore, the best path $p_{b}$ with the optimal fitness fitness $\left(p_{b}\right)$ will be selected.

After that if the number of possible alternative paths in the set $P_{\text {all }}$ is more than 5, the selection of calculated possible alternative paths would be so complex, and we would use the swarm intelligent algorithm to optimize the selection of optimal path, or else the optimal path would be calculated and selected directly. We consider each possible alternative path $p_{j}$ from source $v_{s}^{i}$ to $v_{\text {sink }}$ as a solution, and the number of solutions in the population is $m$. The details of swarm intelligent algorithm are described in the next section.

\section{Design of the Protocol in MSWSN}

3.1. The Swarm Intelligent Algorithm of Route Maintaining Protocol. The kernel optimization algorithm of our proposed route maintaining protocol is a swarm intelligent optimization algorithm. This optimization algorithm would compute and select the optimized path from source node to the sink among all the possible paths, in which each possible path represents a solution. As new avenues in the field of optimization, swarm intelligence was defined by Bonabeau as the attempt to design algorithms or distributed problemsolving devices inspired by collective behavior of social insect colonies and other animal societies [16]. Common examples range from flock of birds to colony of bees. The main essence of swarm intelligence is including the functional behavior of these group agents into a practical computational model [13], such as artificial bee colony (ABC) algorithm.

The population-based artificial bee colony (ABC) algorithm proposed by Okdem et al. is based on the minimal foraging model of honey bees used in nectar collection from the adjoining environment of their honeycomb [17], with the behavior like self-organization, task allocation, and communication among the individuals. Its advantages of fewer parameter settings, faster convergence speed, and higher convergence precision have attracted the attention of many scholars since it was proposed. The algorithm has been successfully applied in the function optimization problem, WSN, and other areas $[18,19]$. The tests for several standard functions by [14] have demonstrated that the performance of $\mathrm{ABC}$ is better than the mainstream optimization algorithms, such as GA, PSO, and DE algorithm.

In the $\mathrm{ABC}$, bees are categorized into three groups: employed bees, onlooker bees, and scout bees. The employed bees search food sources and share the information to recruit the onlooker bees. The onlooker bees make decision to choose a food source from those found by the employed bees and search food around it. The food source that has more nectar amount (fitness value) would have a higher probability to be selected by onlooker bees. The scout bees are translated from a few employed bees, which discard their food sources and randomly search new ones. For a search problem in a $D$ dimensional space, the position of a food source represents a potential solution. The nectar amount of a food source is the fitness value of the associate solution. Each food source is exploited by only one employed bee. The number of employed or onlooker bees is equal to the number of solutions in the population.

The ABC can use the positive feedback mechanisms of optimized search between bees to effectively speed up the process of global optimization and set fewer parameters. But when searching in the near global optimal solution, the search speed would slow down and the diversity of population would be reduced. Therefore, we draw on outstanding diversity characteristic of immune mechanism and develop the IABC algorithm. Each solution would be considered as an antibody. After the antibody clone and selection step, the better one is reserved and the worse one is discarded, which would increase its diversity.

3.1.1. Artificial Bee Colony Mechanism. $X_{i}=x_{i 1}, x_{i 2}, \ldots, x_{i D}$ is the $i$ th food source (solution) in the population, and $D$ is the problem dimension size. Each employed bee generates a new food source $V_{i}$ around the neighborhood of its previous food source position as follows:

$$
v_{i j}=x_{i j}+\phi_{i j}\left(x_{i j}-x_{k j}\right),
$$

where $i=1,2,3, \ldots, \mathrm{SN}, \mathrm{SN}$ is the population size, $j=$ $1,2, \ldots, D$ is a random index, and $x_{k}$ is a randomly selected solution in the current population $(k \neq i) . \phi_{i j}$ is a random number in the range $[-1,1]$. If the new $V_{i}$ is better than its parent $X_{i}$, then $V_{i}$ replaces $X_{i}$.

After employed bees phase, the probability value $\mathrm{Pro}_{i}$ is calculated according to the food sources fitness as the following:

$$
\operatorname{Pro}_{i}=\frac{\text { fitness }\left(x_{i}\right)}{\sum_{i=1}^{\mathrm{SN}} \text { fitness }\left(x_{i}\right)},
$$

where fitness $\left(x_{i}\right)$ is the fitness value of the $i$ th solution in the population and $\mathrm{Pro}_{i}$ is proportional to fitness $\left(x_{i}\right)$. A better food source has higher probability to be selected. 
If a food source cannot be improved further over a predefined number of rounds, the food source is abandoned. Assume that the abandoned source is $x_{i}$; the scout bee randomly searches a new food source to be replaced with $x_{i}$. The operation is defined as follows:

$$
x_{i j}=x_{j}^{\min }+\operatorname{Rand}(0,1)\left(x_{j}^{\max }-x_{j}^{\min }\right),
$$

where $\operatorname{Rand}(0,1)$ is uniformly distributed in the range $[0,1]$ and $\left[x_{j}^{\min }, x_{j}^{\max }\right]$ is the boundary constraint for the $j$ th variable. Then, if the fitness value of the solution is the optimal fitness or the number of iterations increases from zero to Gen, the optimal path $p_{\text {op }}$ (solution) would be output, or else go to the immunization step.

3.1.2. Immunization Mechanism. In this step, each solution would be considered as an antibody. The sequence number SN of solution $x_{i}$ arranged in the optimal solution set $X_{b}$ is considered as the affinity of solution $\mathrm{SA}_{i}$, and $\mathrm{SA}_{i}=\mathrm{SN}$. Then, the clone number $\mathrm{CN}_{i}$ is calculated as follows:

$$
\mathrm{CN}_{i}=\mathrm{SA}_{i} \times \frac{N_{p}}{\left\lceil\sum_{j=1}^{m} j\right\rceil}
$$

where $N_{p}$ is the solution number, $m$ is the size of optimal solution set $X_{b}$, and the total cloned solution number is Sum $=\sum_{j=1}^{m} \mathrm{CN}_{i}$. Thus, the cloned solution number is proportional to the fitness. Then, the solution mutation is used in the clone populations and the mutation rule is as follows:

$$
\mathrm{CS}_{i}=x_{i}+\gamma \operatorname{Rand}(0,1) \text {, }
$$

where $x_{i}$ is the original antibody, $\gamma$ represents mutation factors and $\gamma=0.5$, and $\mathrm{CS}_{i}$ is the clone solution individual. In the solution restrain rule, we calculate the antigen stimulus degree of solution in $N\left(X_{b}\right)$ and the mutation solution. The Euclidean distance between solution $N\left(\mathrm{CS}_{t}\right)$ and antigen (fitness) fitness $\left(x_{t}\right)$ is $D(i, j)=\sqrt{\sum_{i=1}^{n}\left(\mathrm{CS}_{i t}-\operatorname{fitness}\left(x_{i t}\right)\right)^{2}}$.

Therefore, the stimulus degree of antibody (solution) is

$$
\operatorname{SD}(i, j)=\frac{1}{D(i, j)}=\frac{1}{\sqrt{\sum_{i=1}^{n}\left(\mathrm{CS}_{i t}-\text { fitness }\left(x_{i t}\right)\right)^{2}}} .
$$

After comparing each solution with the stimulus threshold $\mathrm{Th}$, the better solution $(\mathrm{SD}(i, j)>\mathrm{Th})$ would be reserved in the memory cell, and the worse one is discarded. The detailed process of the proposed IABC algorithm is shown in Figure 3.

The IABC optimization algorithm is the kernel algorithm of our route maintaining protocol of the MSWSN. To deal with the path maintaining problem due to the movement of sink, the proposed algorithm would optimize the path fitness function to provide the fast routing recovery mechanism with an alternative optimal-fitness path. Apparently, the more suitable alternative path selected from source to the mobile sink would contain nodes with stronger signal strength, less end-to-end delay, and higher battery voltage.
3.2. Assumption of Energy Model. The calculation of minimum energy consumption emphasizing the effect of distances will be as in (8) and (9) expressing sum of the energy consumptions of network [9]. The abbreviations $j$, Ene $_{j}$, Ene $^{\mathrm{TX}}, \mathrm{Ene}^{\mathrm{RX}}$, Ene $\mathrm{Elec}^{\mathrm{el}}$, Ene ${ }^{\mathrm{amp}}, k$, and $d$ in (9) are node index, energy consumption of the $j$ th node, transmit energy, receive energy, radio electronics parameter, transmit amplifier parameter, number of bits of the transmitting data, and distance value between $j$ th node and next-hop node, respectively:

$$
\begin{aligned}
\text { Ene }_{j} & \geq\left(\text { Ene }^{\mathrm{RX}}+\text { Ene }^{\mathrm{TX}}\right) \\
\text { Ene }^{\mathrm{RX}} & =\text { Ene }^{\text {elec }} \cdot k, \\
\text { Ene }^{\mathrm{TX}} & =\left(\text { Ene }^{\text {elec }}+\text { Ene }^{\mathrm{amp}} \cdot d_{j}^{2}\right) \cdot k .
\end{aligned}
$$

Using this method, the total energy consumption of the data transmission and executing the proposed IABC per round can be calculated in the simulation.

\section{Experimental Evaluation of the Route Maintaining Protocol}

4.1. Model and Assumption. Our system uses MATLAB 2008a to simulate and evaluate the performance of the protocol. The experimental hardware environments are Intel i7$4600 \mathrm{M}, 2.90 \mathrm{GHz}$ CPU, and $4 \mathrm{~GB}$ memory, and the operating system is MS windows 7. The whole MSWSN is simulated in the area of $3500 \mathrm{~m} \times 3500 \mathrm{~m}$. The field is static and 200 300 sensor nodes are deployed uniformly in which $10 \%$ sensor nodes are source nodes. The sensor nodes are homogeneous and have the same initial energy of $120 \mathrm{~J}$. Their communication radius is $300 \mathrm{~m}$. This experiment compares our protocol with TTDD, ERTLD routing protocols for MSWSN. The purpose of the simulation is to illustrate that our protocol could provide a more robust and efficient transmission environment.

The other network environment parameters are as follows: One mobile sink moves in the network and its speed is $3 \sim 6 \mathrm{~m} / \mathrm{s}$, the source node delivers packets at the rate of 20 data packets per round, with $10 \mathrm{~KB}$ of each packet size, and the simulation lasts for 600 rounds. In the energy model, Ene $^{\text {elec }}=40 \mathrm{~nJ} / \mathrm{bit}$ and Ene $\mathrm{Emp}^{\mathrm{amp}} 60 \mathrm{~nJ} / \mathrm{bit}$. The sink is assumed to provide sufficient energy to receive data from nodes and operate our protocol. The values of parameters used for the IABC are function dimension $D=20$ and iterated generation $\mathrm{Gen}=150$.

A snapshot from the source node to the mobile sink during the network simulation is shown in Figure 4. We can see that when the sink moves from A to a new coordinate $\mathrm{B}$, the source node immediately establishes an optimal alternative path (path 1-2-3-8-9-10-11-B in Figure 4) to reach the sink, so as to replace the previous broken path (path 1-2-3-4-5-6-7-A in Figure 4).

4.2. Evaluation of the Experimental Results. The performance metrics used for the comparison are packet delivery ratio 


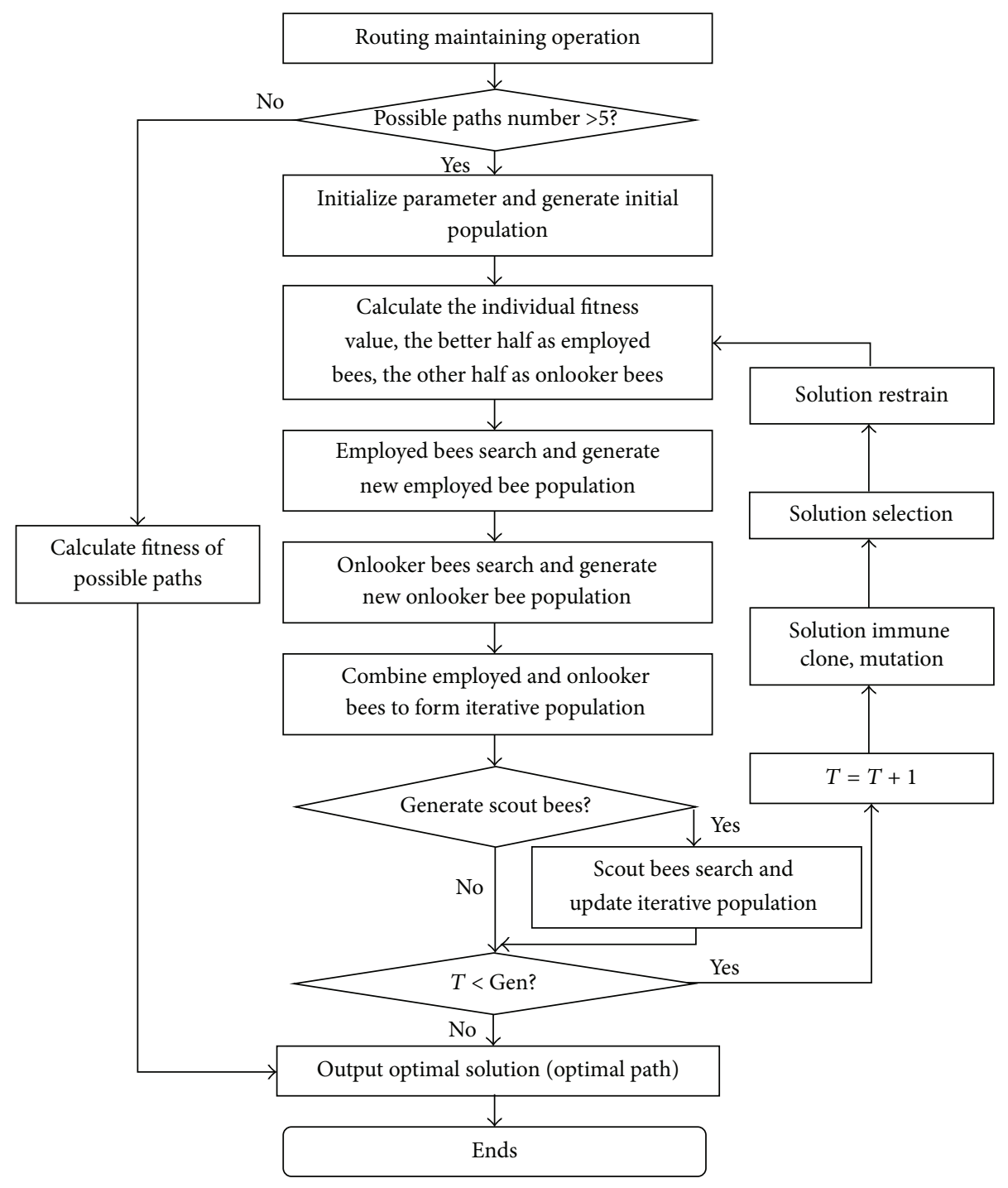

FIGURE 3: The flowchart of the IABC algorithm for route maintaining.

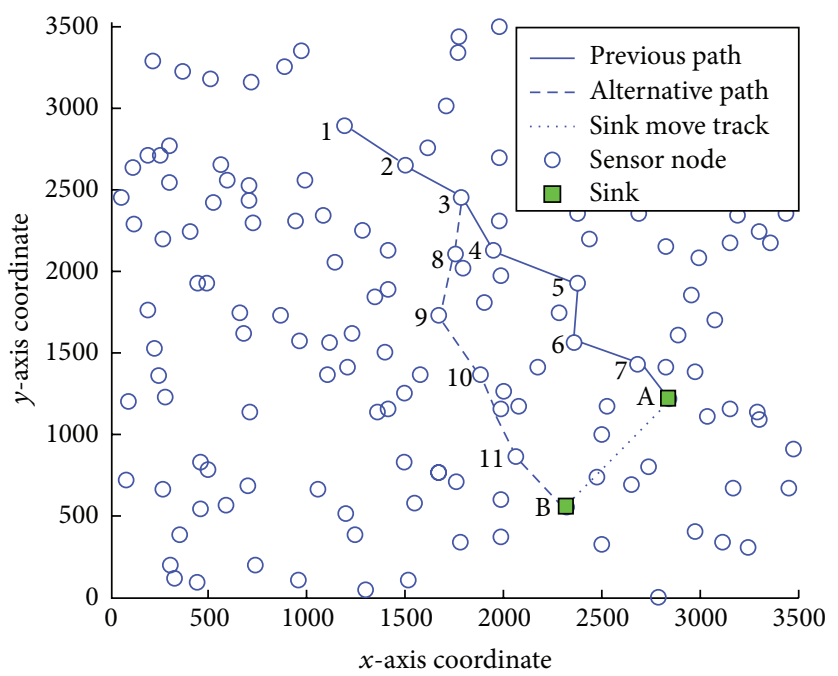

FIGURE 4: The snapshot of network routing simulation with our protocol. 


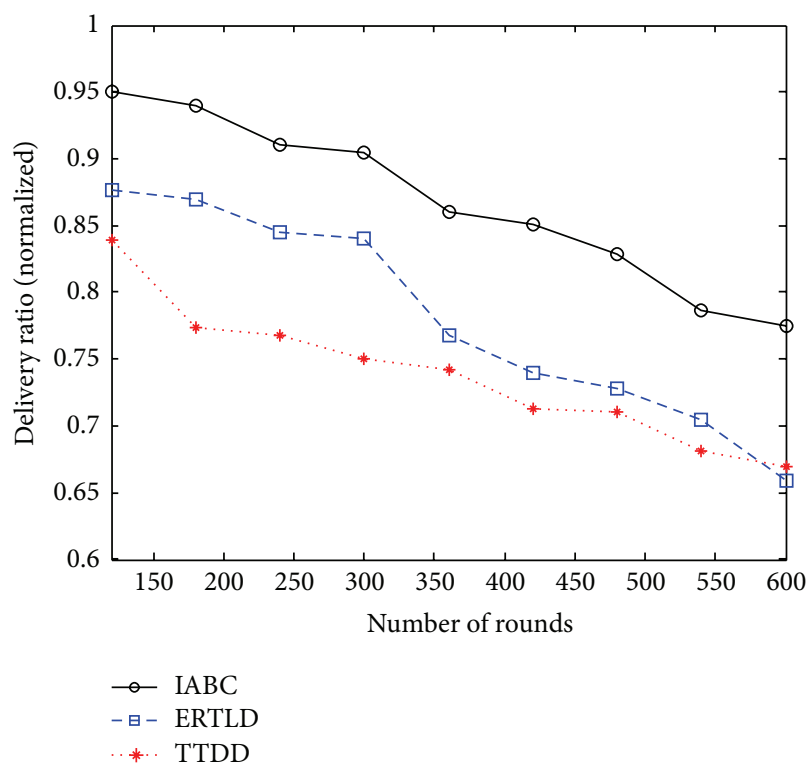

(a) 200 sensor nodes

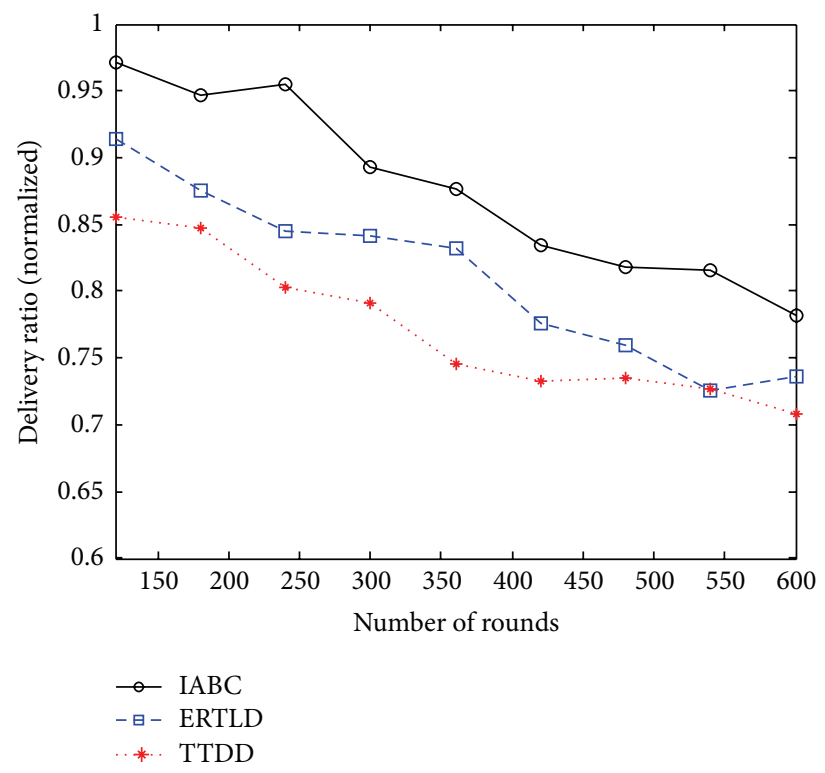

(b) 300 sensor nodes

Figure 5: Comparison of packet delivery ratio between the three protocols with different node number: (a) 200 sensor nodes and (b) 300 sensor nodes.

(the ratio between the successfully received data packets at the sink and the successfully sent data packets by the source node), energy expenditure ratio (the ratio between the consumed energy of nodes and initial energy of these nodes), and average end-to-end delay (the difference between the time a packet is received by the sink and the time it was originally sent by the source node). The results of packet delivery ratio and energy expenditure ratio are normalized, which is helpful to compare their performances.

In terms of packet delivery ratio in Figures 5(a) and 5(b), it can be clearly seen that the delivery ratio for all protocols drops as the number of simulation rounds is increased. This is because the mobility of sink affects the quality of the selected links of the path; the process of links repair and loss of packet would reduce the packet delivery ratio. Among the selected protocols, IABC has the highest packet delivery rate and ERTLD achieves the second one. This is due to several factors: data traffic is routed along shorter paths by using concentric ring mechanism and IABC optimizing algorithm, which would reduce the packet loss rate; the intelligent swarm optimization mechanism keeps the route update faster and more efficiently, with its fast responsiveness to the changing sink position.

In our second experiment presented in Figures 6(a) and $6(\mathrm{~b})$, we vary the velocity of a mobile sink from $3 \mathrm{~m} / \mathrm{s}$ to $6 \mathrm{~m} / \mathrm{s}$. The energy expenditure ratios of all protocols are increased with an increasing number of simulation rounds. This ratio also increases as the mobile sink moves faster, because the change of the frequent topology will incur heavier communication overhead. IABC has lower energy expenditure, followed by ERTLD and finally TTDD. IABC performs slightly better due to its concentric ring mechanism, which can guide the route researching direction and reduce communication overhead of nodes, and also due to the intelligent routing optimization mechanism which enables faster recovery of routes and reduces the energy consumption of protocol. The delivery rate trend in the case of higher velocity of mobile sink is also as expected, dropping with higher velocities. This is due to the fact that nodes would consume more energy to search the route to the sink when they moves faster away from their transmission radius. Notably, the appropriate speed of the sink will be needed for all protocols, which can better reflect the performance of the routing protocols.

In the third experiment, the smaller average end-to-end delay means the faster data transmission. We can observe in Figures $7(\mathrm{a})$ and $7(\mathrm{~b})$ that the IABC outperforms the ERTLD and TTDD in terms of average delay. It can be explained by the faster node communication routing and shorter alternative path selection of the proposed IABC for route maintaining, which can balance the network traffic load and prolong the network lifetime. The delay curve value of IABC almost has not changed as the number of simulation rounds increases, because our protocol would always choose the optimal path with the stable routing delay. Comparing with Figures 7(a) and 7(b), as the more sensor nodes number indicates the longer delivery length of the path, thus the advantage of our IABC with the better alternative path selection is demonstrated more obviously, which means that our routing protocol is more suitable for deploying in the large scale networks with mobile sink.

In summary, these experiments demonstrate clearly the routing optimization ability of IABC and its intelligent optimization mechanism to quickly identify routes to mobile 


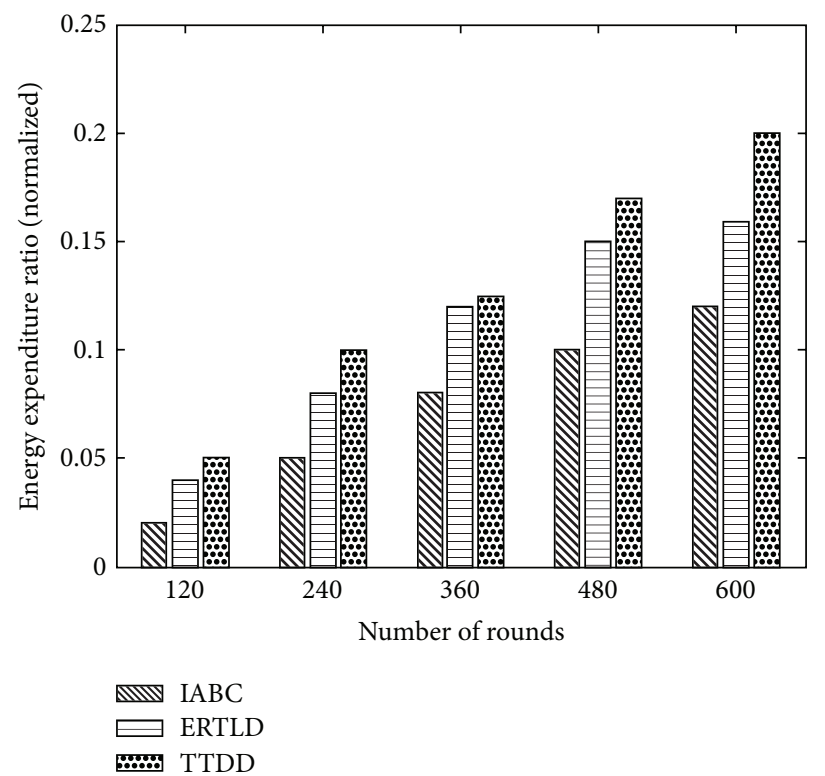

(a) Sink speed $3 \mathrm{~m} / \mathrm{s}$

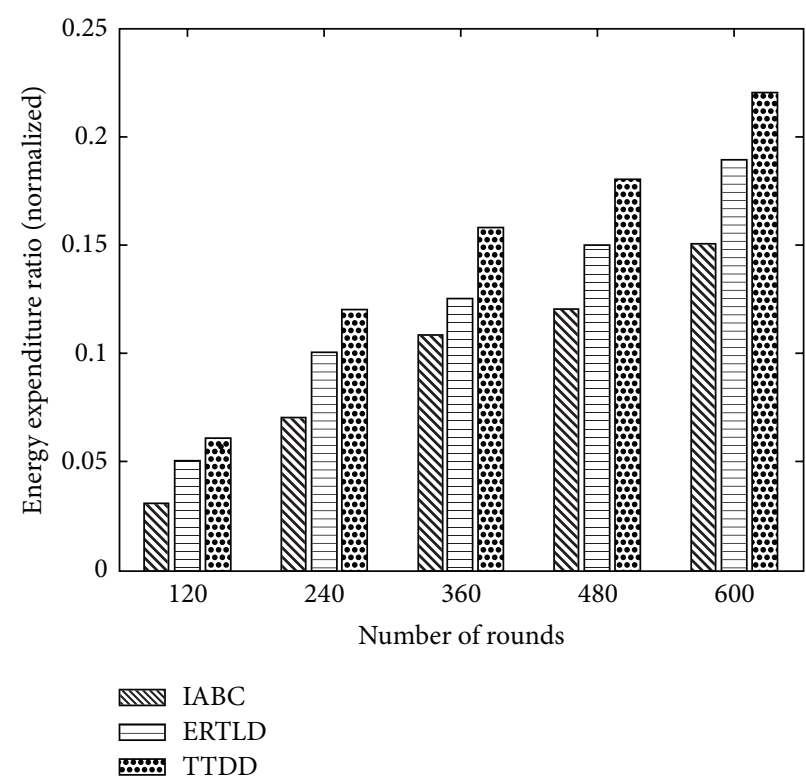

(b) Sink speed $6 \mathrm{~m} / \mathrm{s}$

FIGURE 6: Comparison of energy expenditure between the three protocols as a function of different node speeds: (a) sink speed $3 \mathrm{~m} / \mathrm{s}$ and (b) sink speed $6 \mathrm{~m} / \mathrm{s}$.

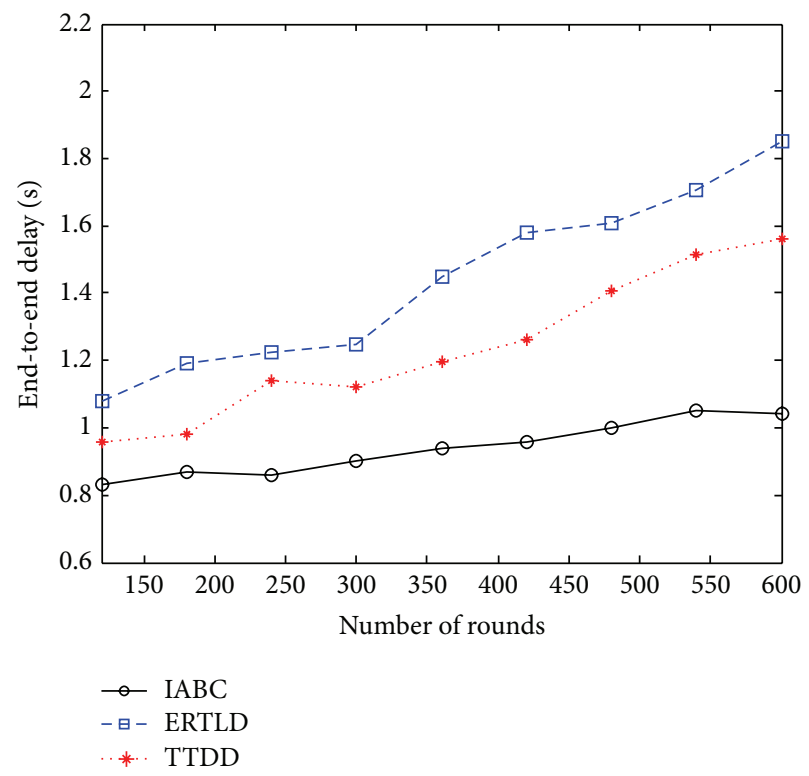

(a) 200 sensor nodes

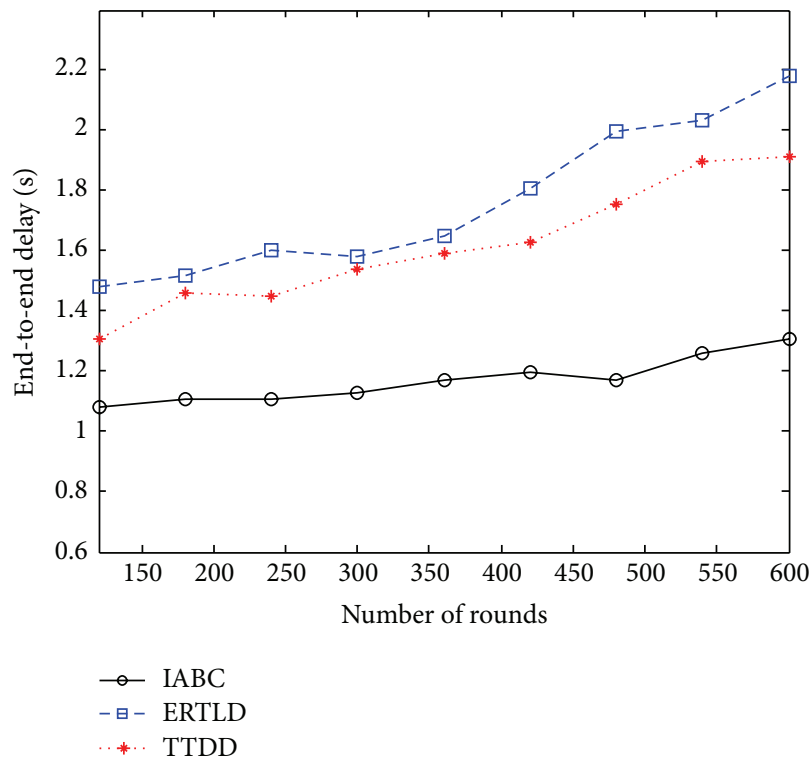

(b) 300 sensor nodes

Figure 7: Comparison of end-to-end delay between the three protocols with different node number: (a) 200 sensor nodes and (b) 300 sensor nodes.

sink. Compared to the other routing protocols, it consumes significantly less energy, needs less end-to-end delay, and achieves considerably higher delivery rates.

\section{Conclusion}

This study presents a novel route maintaining protocol based on the IABC for the MSWSN. In the proposed protocol, the concentric ring mechanism is utilized to guide the route researching direction, and the optimal routing selection is adopted to preserve the data delivery route in the network. Using the immune based artificial bee colony (IABC) algorithm to optimize the forwarding path, the protocol could find an alternative routing path quickly and efficiently when the coordinate of sink is changed in MSWSN. More importantly, this paper demonstrated the applicability and the potential of IABC algorithm for solving routing optimization 
problems. The results of our extending performance are compared to the other aforementioned routing protocols in terms of energy, packet delivery, and delay. Our proposed route maintaining protocol could efficiently solve the energy hole problem, balance the network traffic load, and maintain the network robustness against topology changes.

In the future we will focus on improving the convergence performance, reducing the computational complexity of the IABC algorithm, and validating the proposed protocol on different scenarios with various movement trajectories of mobile sink, and the most important optimization objective is maximizing the network lifetime. In addition, the nodes would have GPS to locate themselves, and the sink may broadcast its position instead of CR packets to build the concentric ring mechanism.

\section{Conflict of Interests}

The authors declare that there is no conflict of interests regarding the publication of this paper.

\section{Acknowledgments}

This work was supported in part by the National Natural Science Foundation of China (nos. 61401257 and 61501282), Shandong Province Young and Middle-Aged Scientists Research Awards Fund (nos. BS2013DX021 and BS2013DX019), Shandong Academy Young Scientists Fund Project (no. 2013QN037), Shandong Academy of Science Doctoral Fund (no. [2012] 58), Shandong Academy Basic Research Fund, and Shandong Academy of Sciences Pilot Project for Science and Technology.

\section{References}

[1] H. W. Ding, D. F. Zhao, Y. S. Gao, and N. S. Zhao, "Research progress of wireless sensor network," Experiment Science \& Technology, vol. 9, no. 6, pp. 26-29, 2011.

[2] Y. S. Jiang, W. R. Shi, X. G. Wang, and H. B. Li, "A distributed routing for wireless sensor networks with mobile sink based on the greedy embedding," Ad Hoc Networks, vol. 20, pp. 150-162, 2014.

[3] A. Jawahar, S. Radha, and S. Vadivelan, "Connectivityguaranteed hybrid topology management scheme for improving the operational lifetime of 3-dimensional wireless sensor networks," International Journal of Distributed Sensor Networks, vol. 2010, Article ID 547368, 11 pages, 2010.

[4] Y. Xue, X. M. Chang, S. M. Zhong, and Y. Zhuang, "An efficient energy hole alleviating algorithm for wireless sensor networks," IEEE Transactions on Consumer Electronics, vol. 60, no. 3, pp. 347-355, 2014.

[5] J. Wang, X. Q. Yang, Z. Q. Zhang, B. Li, and J.-U. Kim, "A survey about routing protocols with mobile sink for wireless sensor network," International Journal of Future Generation Communication and Networking, vol. 7, no. 5, pp. 221-228, 2014.

[6] K. Karenos and V. Kalogeraki, "Traffic management in sensor networks with a mobile sink," IEEE Transactions on Parallel and Distributed Systems, vol. 21, no. 10, pp. 1515-1530, 2010.

[7] Y.-F. Hu, Y.-S. Ding, L.-H. Ren, K.-R. Hao, and H. Han, "An endocrine cooperative particle swarm optimization algorithm for routing recovery problem of wireless sensor networks with multiple mobile sinks," Information Sciences, vol. 300, pp. 100113, 2015.

[8] A. Förster and A. L. Murphy, "Froms: a failure tolerant and mobility enabled multicast routing paradigm with reinforcement learning for WSNs," Ad Hoc Networks, vol. 9, no. 5, pp. 940-965, 2011.

[9] H. Luo, F. Ye, J. Cheng, S. Lu, and L. Zhang, "TTDD: twotier data dissemination in large-scale wireless sensor networks," Wireless Networks, vol. 11, no. 1-2, pp. 161-175, 2005.

[10] S. Tian, S. M. Shatz, Y. Yu, and J. Li, "Querying sensor networks using Ad Hoc mobile devices: a two-layer networking approach," Ad Hoc Networks, vol. 7, no. 5, pp. 1014-1034, 2009.

[11] H. S. Kim, T. F. Abdelzaher, and W. H. Kwon, "Dynamic delayconstrained minimum-energy dissemination in wireless sensor networks," ACM Transactions on Embedded Computing Systems, vol. 4, no. 3, pp. 679-706, 2005.

[12] A. Ali Ahmed, "An enhanced real-time routing protocol with load distribution for mobile wireless sensor networks," Computer Networks, vol. 57, no. 6, pp. 1459-1473, 2013.

[13] Y. F. Hu, Y. S. Ding, K. R. Hao, L. H. Ren, and H. Han, "An immune orthogonal learning particle swarm optimisation algorithm for routing recovery of wireless sensor networks with mobile sink," International Journal of Systems Science, vol. 45, no. 3, pp. 337-350, 2014.

[14] D. Karaboga and B. Akay, "A comparative study of artificial bee colony algorithm," Applied Mathematics and Computation, vol. 214, no. 1, pp. 108-132, 2009.

[15] D.-T. Ho, J. Park, and S. Shimamoto, "Performance evaluation of the PFSC based MAC protocol for WSN employing UAV in rician fading," in Proceedings of the IEEE Wireless Communications and Networking Conference (WCNC '11), pp. 55-60, Cancún, Mexico, March 2011.

[16] G. J. Zhang and J. W. Hu, "Adaptive distributed gateway discovery with swarm intelligence in hybrid wireless networks," International Journal of Future Generation Communication and Networking, vol. 7, no. 5, pp. 137-150, 2014.

[17] S. Okdem, D. Karaboga, and C. Ozturk, "An application of wireless sensor network routing based on Artificial Bee Colony algorithm," in Proceedings of the IEEE Congress of Evolutionary Computation (CEC '11), pp. 326-330, New Orleans, La, USA, June 2011.

[18] R.-C. Chen, W.-L. Chang, C.-F. Shieh, and C. C. Zou, "Using hybrid artificial bee colony algorithm to extend wireless sensor network lifetime," in Proceedings of the 3rd International Conference on Innovations in Bio-Inspired Computing and Applications (IBICA '12), pp. 156-161, IEEE, Kaohsiung, Taiwan, September 2012.

[19] M. Saleem, G. A. D. Caro, and M. Farooq, "Swarm intelligence based routing protocol for wireless sensor networks: survey and future directions," Information Sciences, vol. 181, no. 20, pp. 4597-4624, 2011. 


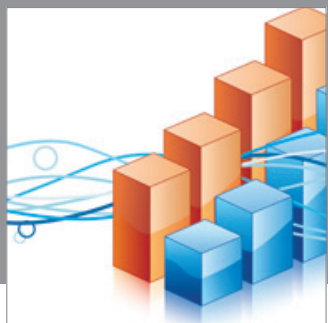

Advances in

Operations Research

mansans

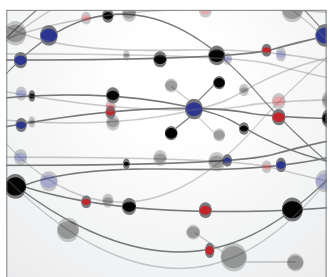

The Scientific World Journal
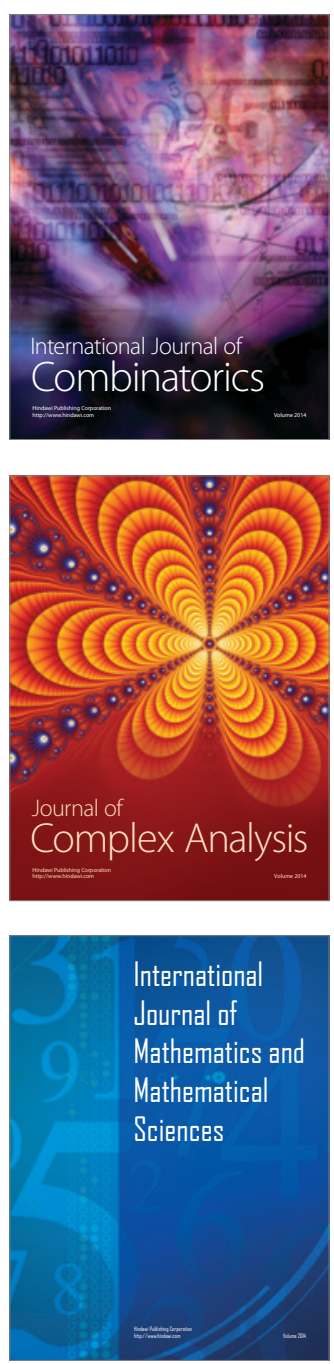
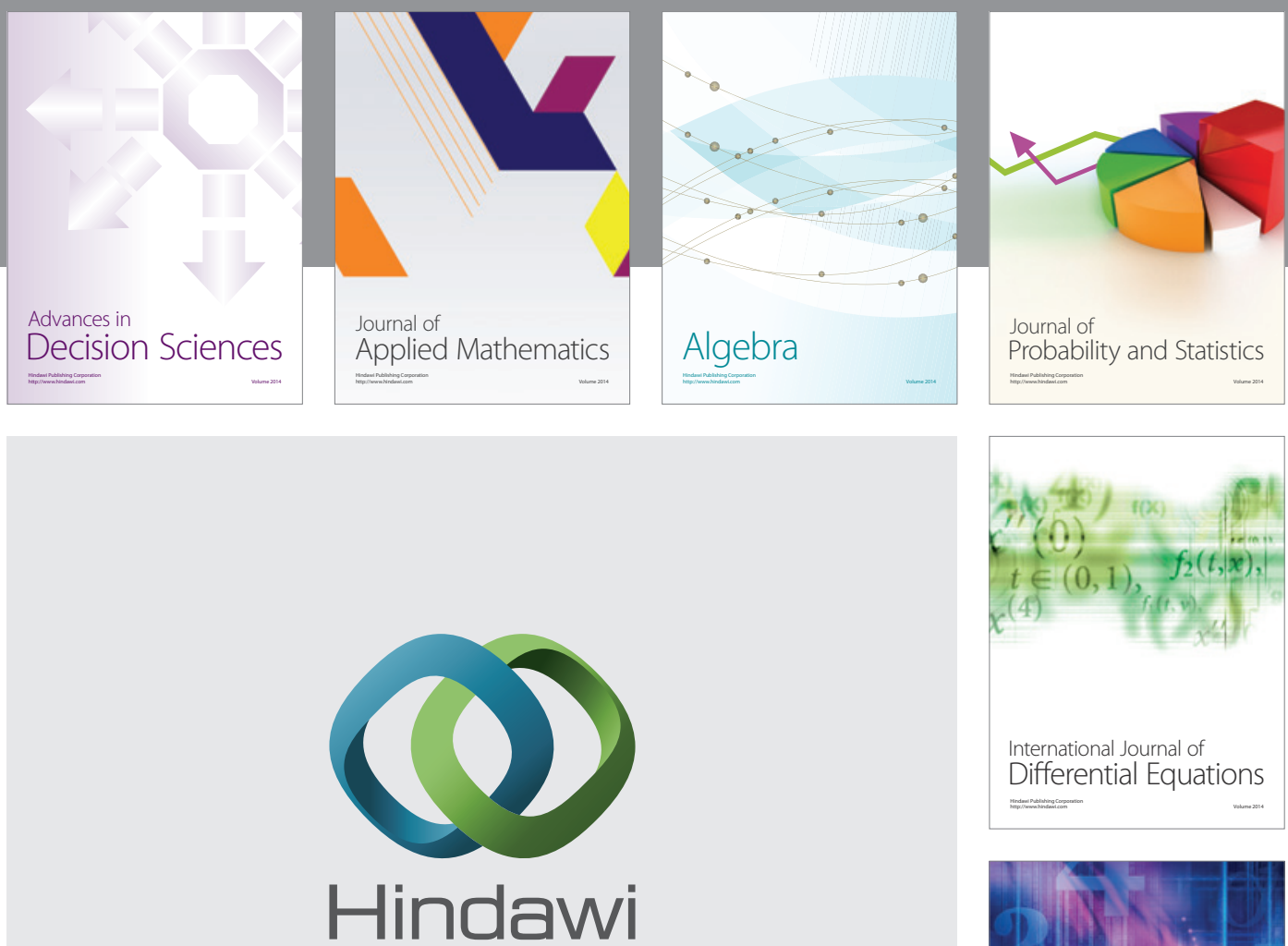

Submit your manuscripts at http://www.hindawi.com
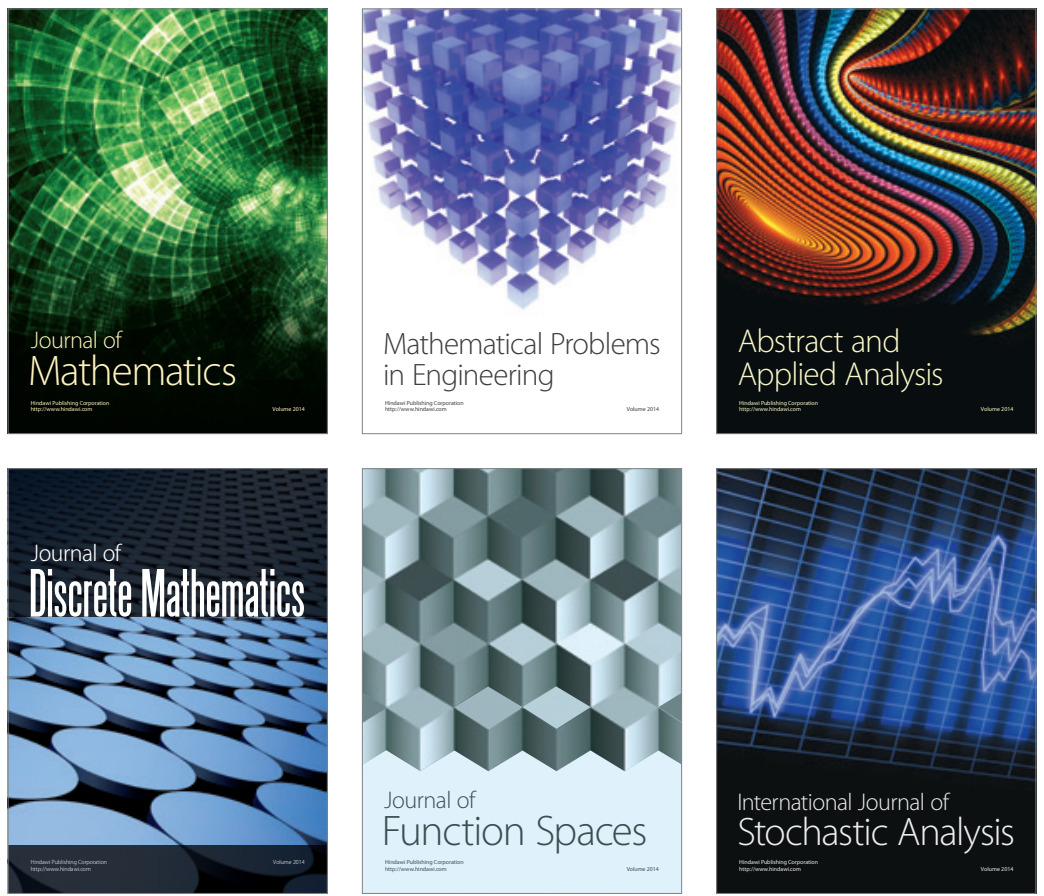

Journal of

Function Spaces

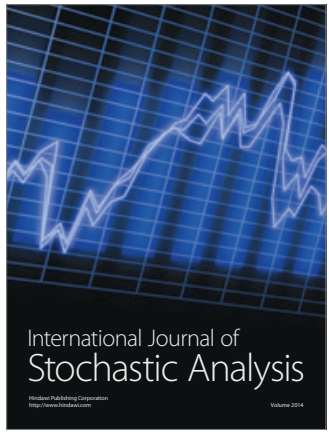

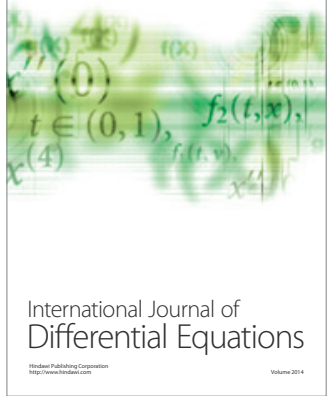
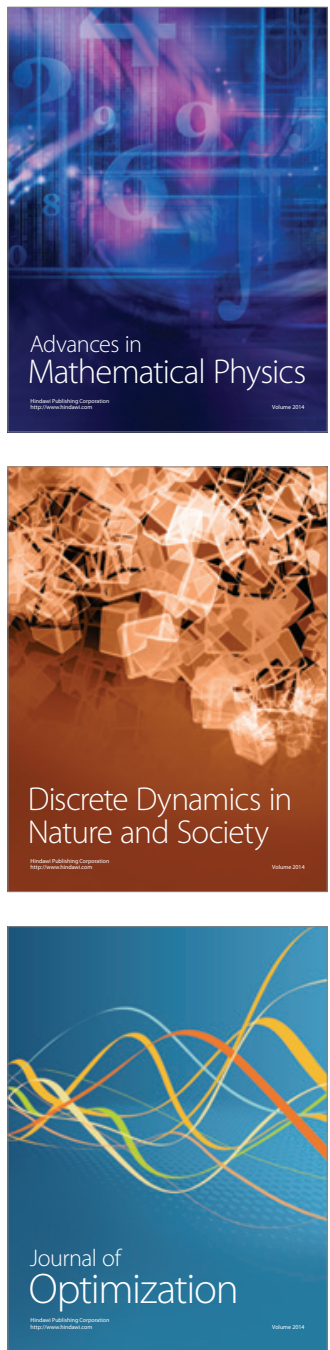